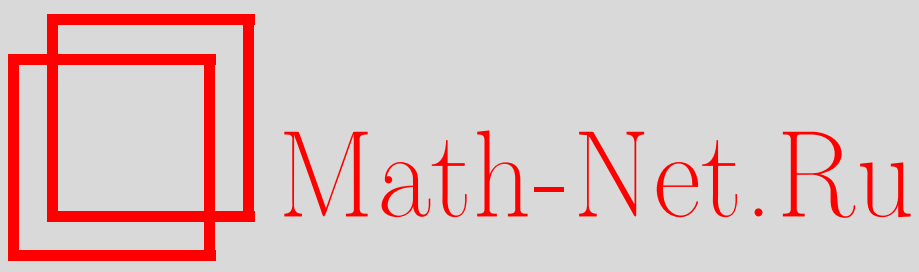

S. Z. Djamalov, S. G. Pyatkov, On some boundary value problems for multidimensional higher order equations of mixed type, Sibirsk. Mat. Zh., 2020, Volume 61, Number 4, 777-795

DOI: https://doi.org/10.33048/smzh.2020.61.405

Use of the all-Russian mathematical portal Math-Net.Ru implies that you have read and agreed to these terms of use http://www . mathnet.ru/eng/agreement

Download details:

IP: 54.172 .240 .79

April 26, 2023, 14:16:10 
Сибирский математический журнал Июль-август, 2020. Том 61, № 4

УДК 517.95

\title{
О НЕКОТОРЫХ КЛАССАХ КРАЕВЫХ ЗАДАЧ ДЛЯ МНОГОМЕРНЫХ УРАВНЕНИЙ СМЕШАННОГО ТИПА ВЫСОКОГО ПОРЯДКА
}

\author{
С. З. Джамалов, С. Г. Пятков
}

\begin{abstract}
Аннотация. Описаны постановки краевых задач общего вида для многомерного уравнения смешанного типа высокого порядка. Приведен способ построения краевых условий, обеспечивающих коэрцитивность задачи. Доказывается обобщенная и регулярная разрешимость поставленных краевых задач в пространствах С. Л. Соболева.

DOI 10.33048/smzh.2020.61.405

Ключевые слова: уравнение смешанного типа, нелокальная краевая задача, краевая задача, обобщенное решение, регулярное решение, единственность решения, существование решения.
\end{abstract}

\section{1. Введение}

Рассматривается вопрос о разрешимости краевых задач для уравнения смешанного типа высокого порядка вида

$$
L u=P u+M u=f(x, t), \quad(x, t) \in Q=G \times(0, T),
$$

где $G \in \mathbb{R}^{n}$ - ограниченная область с границей $\Gamma, S=\Gamma \times(0, T)$,

$$
P u=\sum_{i=0}^{2 n} k_{i}(x, t) u^{(i)}, \quad M u=(-1)^{m} \sum_{|\alpha|,|\beta| \leq m} D^{\alpha}\left(a_{\alpha, \beta}(x) D^{\beta} u\right), \quad u^{(i)}=\frac{\partial^{i} u}{\partial t^{i}} .
$$

Краевая задача. Найти решение уравнения (1), удовлетворяющее краевым условиям

$$
\begin{gathered}
U_{i} u=\sum_{j=0}^{2 n-2}\left(\alpha_{i, j} u^{(j)}(0)+\beta_{i, j} u^{(j)}(T)\right)=0, \quad i=1,2, \ldots, 2 n-1, \\
\left.B_{j} u\right|_{S}=\left.\sum_{|\alpha| \leq m_{j}} b_{j, \alpha}(x) D^{\alpha} u\right|_{S}=0, \quad j=1,2, \ldots, m .
\end{gathered}
$$

В работах М. А. Лаврентьева, И. Н. Векуа, С. А. Христиановича, С. А. Чаплыгина, К. Г. Гудерлея и др. отмечена важность изучения краевых задач для уравнений смешанного типа в связи с задачами, возникающими в трансзвуковой газовой динамике, в безмоментной теории оболочек с кривизной переменного знака и во многих прикладных задачах механики. В исследованиях Фикеры,

Работа выполнена при финансовой поддержке Российского фонда фундаментальных исследований (код проекта 18-51-41009).

(c) 2020 Джамалов С. З., Пятков С. Г. 
О. А. Олейник, Е. В. Радкевича и ряда других авторов (см. библиографию в [1]) предложены новые подходы и методы для построения единой теории краевых задач для эллиптико-параболических уравнений. В. Н. Враговым $[2,3]$ начато построение общей теории краевых задач для уравнений смешанного типа второго и высокого порядков с произвольным многообразием изменения типа, в частности, для гиперболо-параболических уравнений. Довольно много работ посвящено краевым задачам для уравнений смешанного типа второго порядка (см. [4-11]). Работ, посвященных уравнениям высокого порядка, не так много. Опишем известные результаты. Сошлемся прежде всего на [12], где изучаются краевые задачи для уравнения (1) с краевыми условиями вида

$$
\begin{gathered}
\left.\frac{\partial^{k} u}{\partial n^{k}}\right|_{S}=0, k=0,1,2, \ldots, m-1, \quad u^{(i)}(0, x)=0, i=0,1, \ldots, n-1, \\
u^{(i)}(T, x)=0, i=1, \ldots, n-1,\left.\quad u^{(n)}\right|_{P_{0}^{+}}=0,\left.\quad u^{(n)}\right|_{P_{T}^{-}}=0
\end{gathered}
$$

и вида

$$
\begin{gathered}
\left.\frac{\partial^{k} u}{\partial n^{k}}\right|_{S}=0, k=0,1,2, \ldots, m-1, \quad u^{(i)}(0, x)=0, i=0,1, \ldots, n-2, \\
u^{(i)}(T, x)=0, i=0, \ldots, n-1,\left.\quad u^{(n-1)}\right|_{P_{0}^{+}}=0,\left.\quad u^{(n)}\right|_{P_{T}^{-}}=0,
\end{gathered}
$$

где $P_{0}^{+}=\left\{(x, 0):(-1)^{n-1} k_{2 n}(x, 0)>0\right\}, P_{T}^{-}=\left\{(x, 0):(-1)^{n-1} k_{2 n}(x, 0)<0\right\}$. В [12] предполагается, что оператор $M$ положительно определен. При выполнении естественных условий на коэффициенты получены теоремы существования и единственности обобщенных решений задачи $(1),(4)$ класса $L_{2}\left(0, T ; W_{2}^{m}(G)\right) \cap$ $W_{2}^{n}\left(0, T ; L_{2}(G)\right)$. В случаях, когда $k_{i}=k_{i}(t)$ для всех $i, k_{2 n}(0), k_{2 n}(T) \neq 0$ и $f \in W_{2}^{1}\left(0, T ; L_{2}(G)\right), f(T, x)=0$, доказано существование решений этой задачи из класса $L_{2}\left(0, T ; W_{2}^{2 m}(G)\right) \cap W_{2}^{2 n}\left(0, T ; L_{2}(G)\right)$. Для задачи $(1),(5)$ аналогично доказываются существование и единственность обобщенных решений, в некоторых случаях получена теорема существования регулярных решений, точнее, при условии $(-1)^{n-1} k_{2 n}(0, x)>0,(-1)^{n-1} k_{2 n}(T, x)>0$ в $G$. В этом случае рассмотрен вопрос и о повышении гладкости решений. Широкий класс локальных краевых условий, входящих в класс (2), рассмотрен в [12] при естественных условиях и предположении, что $k_{2 n}=k_{2 n}(t), k_{2 n-1}=k_{2 n-1}(t)$ и величины $k_{2 n}(0), k_{2 n}(T)$ одного знака. Доказаны теоремы о существовании и единственности решений. В данной работе исследуются вопросы существования и единственности решений краевых задач (1)-(3) при других условиях на коэффициент $k_{2 n}$, предполагая, что функции $k_{2 n}(0, x)$ и $k_{2 n}(T, x)$ разных знаков. Также в отличие от работ других авторов класс рассматриваемых краевых задач уже является довольно широким, в частности, он содержит задачи с нелокальными условиями по времени (см. [13]), например, периодические краевые задачи. Кроме того, описан способ построения коэрцитивных краевых условий для обыкновенных дифференциальных операторов нечетного порядка, полученные результаты применяются для для исследования вопросов существования и единственности решений задач (1)-(3). Отметим, что в случае дифференциальных операторов четного порядка и для более общих классов операторов - эллиптических дифференциальных операторов - коэрцитивные краевые условия и способ их построения описаны (см., например, [14, гл. $2, \S 9.4])$. 


\section{2. Вспомогательные результаты}

Положим

$$
\langle u, v\rangle=\int_{G} u(x) \overline{v(x)} d x, \quad(u, v)_{0}=\int_{0}^{T} u(t) \overline{v(t)} d t, \quad(u, v)=\int_{Q} u(x, t) \overline{v(x, t)} d x d t .
$$

Нормы в $L_{2}(0, T)$ и $L_{2}(Q)$ обозначаются через $\|\cdot\|_{0}$ и $\|\cdot\|$. Пусть $E-$ банахово пространство. Через $L_{p}(G ; E)\left(G\right.$ - область в $\left.\mathbb{R}^{n}\right)$ обозначим пространство сильно измеримых функций, определенных на $G$, со значениями в $E$ и конечной нормой \|\|$u(x)\left\|_{E}\right\|_{L_{p}(G)}[15]$. Определение пространств Соболева $W_{p}^{s}(G ; E)$ также стандартное (см. [15-17]). Пусть $D(M)=\left\{u \in W_{2}^{2 m}(G):\left.B_{j} u\right|_{\Gamma}=0, j=\right.$ $1,2, \ldots, m\}$. Далее предполагаем, что

(A) $\Gamma \in C^{2 m}$, оператор $M$ самосопряжен в $L_{2}(G)$ и найдется число $\lambda_{0} \geq 0$ такое, что $\left\langle M u+\lambda_{0} u, u\right\rangle \geq \delta\|u\|_{W_{2}^{m}(G)}^{2}$ для всех $u \in D(M)$, где $\delta>0$ - некоторая постоянная. Считаем, что выполнены условия

$$
\begin{gathered}
a_{\alpha, \beta} \in C(\bar{G})(|\alpha|=|\beta|=m), \quad a_{\alpha, \beta} \in W_{\infty}^{|\alpha|}(G)(|\alpha|+|\beta| \leq 2 m) ; \\
b_{j, \alpha}(x) \in C^{2 m-m_{j}}(\Gamma) \quad\left(|\alpha| \leq m_{j}, j=1,2, \ldots, m\right) .
\end{gathered}
$$

Пусть $k_{j}$ - порядок граничного оператора $U_{j}$. Без ограничения общности считаем, что $k_{1} \leq k_{2} \leq \cdots \leq k_{2 n-1}<2 n-1$, причем $k_{j}<k_{j+2}$ для всех $j$, иначе проведем процедуру нормировки краевых условий (см. [18, гл. 2, § 4, п. 7]). Далее предполагаем, что векторы $\overrightarrow{R_{i}}=\left(\alpha_{i, 0}, \alpha_{i, 2}, \ldots, \alpha_{i, 2 n-2}, \beta_{i, 0}, \beta_{i, 2}, \ldots, \beta_{i, 2 n-2}\right)$, $i=1,2, \ldots, 2 n-1$, отвечающие граничным операторам $(2)$, линейно независимы. Выделим граничные условия $U_{j}$ с $k_{j} \leq n-1$. Пусть это будут условия $U_{1}, \ldots, U_{p}$.

Рассмотрим произвольный оператор $P_{0} u=\sum_{i=0}^{2 n-1} b_{i}(t) u^{(i)}$ и опишем условия его коэрцитивности, т. е. условия существования постоянных $\delta_{0}>0, \lambda_{0}$ таких, что

$$
\operatorname{Re}\left(P_{0} u+\lambda_{0} u, u\right)_{0} \geq \delta_{0}\|u\|_{W_{2}^{n-1}(0, T)}^{2}
$$

для всех $u$ из $D\left(P_{0}\right)$, области определения $P_{0}$, состоящей из функций, принадлежащих $W_{2}^{2 n-1}(0, T)$ и удовлетворяющих $(2)$. Пусть выполнены условия

$$
b_{i} \in W_{2}^{i-n+1}(0, T), i \geq n, \quad b_{i} \in W_{2}^{1}(0, T), i<n, k_{p}=n-1, k_{p-1}<n-1
$$

существует постоянная $\delta_{1}>0$ такая, что

$$
(-1)^{n}\left(b_{2 n-1}^{\prime}(t)(n-1 / 2)-b_{2 n-2}(t)\right) \geq \delta_{1}, \quad\left|b_{2 n-1}(t)\right| \geq \delta_{1} \quad \forall t \in[0, T]
$$

и коэффициенты $b_{2 n-1}, b_{2 n-2}$ вещественны. При выполнении условия (9) для главной части $U_{0 p}$ граничного оператора $U_{p}$ имеем три возможности:

(a) $U_{0 p} u=\alpha_{p, n-1} u^{(n-1)}(0), \alpha_{p, n-1} \neq 0$ и $\beta_{p, n-1}=0$;

(b) $U_{0 p} u=\beta_{p, n-1} u^{(n-1)}(T), \beta_{p, n-1} \neq 0$ и $\alpha_{p, n-1}=0$;

(c) $U_{0 p} u=\alpha_{p, n-1} u^{(n-1)}(0)+\beta_{p, n-1} u^{(n-1)}(T)$, причем $\alpha_{p, n-1} \beta_{p, n-1} \neq 0$.

В первых двух случаях соответственно предположим, что на $(0, T)$

$$
\text { (a) } \quad(-1)^{n-1} b_{2 n-1}(t)>0, \quad \text { (b) } \quad(-1)^{n-1} b_{2 n-1}(t)<0,
$$

и в третьем случае предположим, что

$$
\text { (c) }(-1)^{n-1}\left(b_{2 n-1}(T)-\alpha^{2} b_{2 n-1}(0)\right)>0, \quad \alpha=\beta_{p, n-1} / \alpha_{p, n-1} .
$$


Дополним набор $R_{1}, \ldots, R_{p}$ векторами $\overrightarrow{R_{i}^{\prime}}=\left(\alpha_{i, 0}^{\prime}, \alpha_{i, 1}^{\prime}, \ldots, \alpha_{i, n-1}^{\prime}, \beta_{i, 0}^{\prime}, \beta_{i, 1}^{\prime}, \ldots\right.$, $\left.\beta_{i, n-1}^{\prime}\right), i=p+1, p+2, \ldots, 2 n$, так, чтобы система $R_{1}, R_{2}, \ldots, R_{p}, R_{p+1}^{\prime}, \ldots, R_{2 n}^{\prime}$ была линейно независимой и выполнены условия: система операторов $U_{1}, U_{2}$, $\ldots, U_{p}, U_{p+1}^{\prime}, \ldots, U_{2 n}^{\prime}\left(U_{j}^{\prime}-\right.$ граничные операторы, отвечающие векторам $\left.R_{j}^{\prime}\right)$ нормирована (см. [18, гл. $2, \S 4$, п. 7]) и ord $U_{j}^{\prime} \leq n-1$ для всех $j$. В этом случае порядки граничных операторов пробегают множество $0,1, \ldots, n-1$ и имеется ровно два оператора одного порядка. Среди этих новых операторов обязательно найдется оператор порядка $n-1$ в силу условия (9). Без ограничения общности считаем, что это $U_{p+1}^{\prime}$. Пусть строки матрицы $B$ (начиная с верхней строчки) суть векторы этой системы. Рассмотрим систему уравнений

$$
B \vec{y}=U, \quad U=\left(U_{1}, U_{2}, \ldots, U_{p}, U_{p+1}^{\prime}, \ldots, U_{2 n}^{\prime}\right)^{T}
$$

и найдем ее решение $\vec{y}=\left(y_{1}, \ldots, y_{2 n}\right)$. Для $v(t) \in W_{2}^{2 n-1}(0, T)$ положим $v^{(i)}(0)=$ $y_{i+1}, v^{(i)}(T)=y_{i+n+1}(i \leq n-1)$. Таким образом, получили представления вида

$$
\begin{gathered}
v^{(i)}(0)=\sum_{j=1}^{p} \gamma_{i, j} U_{j} v+\sum_{j=p+1}^{2 n} \gamma_{i, j} U_{j}^{\prime} v, \\
v^{(i)}(T)=\sum_{j=1}^{p} \gamma_{i+n+1, j} U_{j} v+\sum_{j=p+1}^{2 n} \gamma_{i, j+n+1} U_{j}^{\prime} v,
\end{gathered}
$$

где $i=0,1, \ldots, n-1$ и $\gamma_{i, j}$ - элементы матрицы $B^{-1}$. Интегрируя по частям, получим

$$
\begin{aligned}
\left(P_{0} u, v\right)_{0}=\sum_{i=0}^{2 n-1}\left(b_{i} u^{(i)}, v\right)_{0}=\sum_{i=0}^{n-1}\left(b_{i} u^{(i)}, v\right)_{0} & \\
& +\sum_{i=n}^{2 n-1}\left(u^{(n-1)},\left(\bar{b}_{i} v\right)^{(i-n+1)}\right)_{0}(-1)^{i-n+1} \\
& +\left.\sum_{i=n}^{2 n-1} \sum_{k=1}^{i-n+1}(-1)^{k-1} u^{(i-k)}\left(b_{i} \bar{v}\right)^{(k-1)}\right|_{0} ^{T}=a_{0}(u, v)+a_{1}(u, v) .
\end{aligned}
$$

Используя представления (13) в последнем слагаемом $a_{1}(u, v)$ и приводя подобные, получим, что

$$
a_{1}(u, v)=\sum_{i=1}^{p} F_{i} u \overline{U_{i} v}+\sum_{i=1+p}^{2 n} F_{i} u \overline{U_{i}^{\prime} v}
$$

где выражения $F_{i} u$ имеют такой же вид, что и граничные операторы $U_{j} u$, но, конечно, с другими числами $\alpha_{i, j}, \beta_{i, j}$. Из равенства (14) легко увидеть, что выполнено равенство ord $F_{j}=2 n-2-\operatorname{ord} U_{j}^{\prime}, j=p+1, \ldots, 2 n$, ord $F_{j}=2 n-2-\operatorname{ord} U_{j}$, $j=1, \ldots, p$. Приведем последнее условие, гарантирующее коэрцитивность оператора $P_{0}$ при выполнении условий (9)-(12).

(В) Существуют граничные операторы $U_{j}^{\prime}, j=p+1, \ldots, 2 n$, ord $U_{j}^{\prime} \leq n-1$, такие, что векторы $R_{1}, R_{2}, \ldots, R_{p}, R_{p+1}^{\prime}, \ldots, R_{2 n}^{\prime}$ из коэффициентов этих операторов линейно независимы, система граничных операторов $U_{1}, U_{2}, \ldots, U_{p}, U_{p+1}^{\prime}$, $\ldots, U_{2 n}^{\prime}\left(U_{j}^{\prime}-\right.$ граничные операторы, отвечающие векторам $\left.R_{j}^{\prime}\right)$ нормирована и 
справедливо представление

$$
\begin{aligned}
\left(P_{0} u, v\right)_{0}= & \sum_{i=0}^{2 n-1}\left(b_{i} u^{(i)}, v\right)_{0} \\
=\sum_{i=0}^{n-1}\left(b_{i} u^{(i)}, v\right)_{0} & +\sum_{i=n}^{2 n-1}\left(u^{(n-1)},\left(\bar{b}_{i} v\right)^{(i-n+1)}\right)_{0}(-1)^{i-n+1} \\
& +\sum_{i=1}^{p} F_{i} u \overline{U_{i} v}+F_{p+1} u \overline{U_{p+1}^{\prime} v}+\sum_{i=2+p}^{2 n} U_{i-1} u \overline{U_{i}^{\prime} v}
\end{aligned}
$$

для всех $u, v \in W_{2}^{2 n-1}(0, T)$.

Условие $(\mathrm{B})$ гарантирует, что для всех $u, v \in D\left(P_{0}\right)$ имеет место равенство

$$
\left(P_{0} u, v\right)_{0}=\sum_{i=0}^{n-1}\left(b_{i} u^{(i)}, v\right)_{0}+\sum_{i=n}^{2 n-1}\left(u^{(n-1)},\left(\bar{b}_{i} v\right)^{(i-n+1)}\right)_{0}(-1)^{i-n+1}+F_{p+1} u \overline{U_{p+1}^{\prime} v}
$$

Опишем слагаемое $F_{p} u \overline{U_{p} v}+F_{p+1} u \overline{U_{p+1}^{\prime} v}$, входящее в (16). Сравнивая (14), (16) для функций $u, v \in W_{2}^{2 n-1}(0, T)$ таких, что

$$
u^{(i)}(0)=u^{(i)}(T)=v^{(i)}(0)=v^{(i)}(T)=0 \quad \text { при } i \leq n-2,
$$

получим, что для таких функций

$$
F_{p} u \overline{U_{p} v}+F_{p+1} u \overline{U_{p+1}^{\prime} v}=\left.(-1)^{n-1} b_{2 n-1} u^{(n-1)} v^{(n-1)}\right|_{0} ^{T} .
$$

В силу произвольности величин $u^{(n-1)}(0), u^{(n-1)}(T), v^{(n-1)}(0), v^{(n-1)}(T)$ заключаем, что главная часть выражения $F_{p} u \overline{U_{p} v}+F_{p+1} u \overline{U_{p+1}^{\prime} v}$, т. е. слагаемые, содержащие произведения $u^{(n-1)}(0) \overline{v^{(n-1)}(0)}, u^{(n-1)}(0) \overline{v^{(n-1)}(T)}, u^{(n-1)}(T) \overline{v^{(n-1)}(0)}$, $u^{(n-1)}(T) \overline{v^{(n-1)}(T)}$, совпадает с правой частью в (18). Таким образом, имеем представление

$$
F_{p} u \overline{U_{p} v}+F_{p+1} u \overline{U_{p+1}^{\prime} v}=\left.(-1)^{n-1} b_{2 n-1} u^{(n-1)} \overline{v^{(n-1)}}\right|_{0} ^{T}+\sum_{i=1}^{4} \widetilde{F}_{i} u \overline{\widetilde{U}_{i} v},
$$

где $\widetilde{F}_{i}, \widetilde{U}_{i}-$ граничные операторы такие, что

$$
\operatorname{ord} \widetilde{F}_{i}+\text { ord } \widetilde{U}_{i}<2 n-2, \quad \text { ord } \widetilde{F}_{i} \leq n-1, \quad \text { ord } \widetilde{U}_{i} \leq n-1
$$

Теорема 1. Пусть выполнены условия (9)-(12), (В). Тогда оператор $P_{0}$ коэрцитивен и найдется $\lambda_{0}>0$ такое, что для любой функции $f \in L_{2}(0, T)$ и $\lambda \geq \lambda_{0}$ существует единственное решение $u \in W_{2}^{2 n-1}(0, T)$ задачи

$$
P_{0} u+\lambda u=f, \quad U_{i} u=0, \quad i=1,2, \ldots, 2 n-1 .
$$

Если дополнительно $f \in W_{2}^{1}(0, T)$, то $u \in W_{2}^{2 n}(0, T)$.

ДоказАтельство. Для $u \in D\left(P_{0}\right)$ имеем (см. (17))

$$
\begin{aligned}
\left(P_{0} u, u\right)_{0}+(\lambda u, u)_{0}= & \sum_{i=n}^{2 n-1}\left(u^{(n-1)},\left(\bar{b}_{i} u\right)^{(i-n+1)}\right)_{0}(-1)^{i-n+1} \\
& +\sum_{i=0}^{n-1}\left(b_{i} u^{(i)}, u\right)_{0}+F_{p+1} u \overline{U_{p+1}^{\prime} u}+\lambda_{0}\|u\|_{0}^{2} .
\end{aligned}
$$


Выпишем слагаемые со старшими производными в (21):

$$
\begin{aligned}
& J=(-1)^{n}\left(u^{(n-1)},\left(b_{2 n-1} u\right)^{(n)}\right)_{0}-\left(u^{(n-1)},\left(b_{2 n-2} u\right)^{(n-1)}\right)_{0} \\
= & (-1)^{n} \operatorname{Re}\left[\left(u^{(n-1)}, b_{2 n-1} u^{(n)}+n b_{2 n-1}^{\prime} u^{(n-1)}+\ldots\right)_{0}-\left(u^{(n-1)}, u^{(n-1)} b_{2 n-2}+\ldots\right)_{0}\right],
\end{aligned}
$$

где точки обозначают слагаемые, содержащие производные порядка не больше $n-2$. Отсюда

$$
\begin{aligned}
(-1)^{n} \operatorname{Re} J=\operatorname{Re}\left(u^{(n-1)},\left(b_{2 n-1}^{\prime}\left(n-\frac{1}{2}\right)-b_{2 n-2}\right)\right. & \left.u^{(n-1)}+P_{n-2} u\right)_{0} \\
& +\left.\frac{1}{2}\left|u^{(n-1)}\right|^{2} b_{2 n-1}\right|_{0} ^{T},
\end{aligned}
$$

где $P_{n-2}-$ дифференциальный оператор порядка не больше чем $n-2$. Из (10) имеем неравенство

$$
\operatorname{Re} J \geq \delta_{1}\left\|u^{(n-1)}\right\|_{0}^{2}-\left|\left(u^{(n-1)}, P_{n-2} u\right)_{0}\right|+\left.\frac{(-1)^{n}}{2}\left|u^{(n-1)}\right|^{2} b_{2 n-1}\right|_{0} ^{T} .
$$

Рассмотрим граничные слагаемые в (21). Используя (19), получим

$$
\begin{aligned}
F_{p+1} u \overline{U_{p+1}^{\prime} u}=F_{p+1} u \overline{U_{p+1}^{\prime} u}+F_{p} u \overline{U_{p} u} & \\
& =\left.(-1)^{n-1} b_{2 n-1}\left|u^{(n-1)}\right|^{2}\right|_{0} ^{T}+\sum_{i=1}^{4} \widetilde{F}_{i} u \overline{\widetilde{U}_{i} u} .
\end{aligned}
$$

Используя (21), (24) и (25), имеем

$$
\begin{aligned}
J_{2}=\operatorname{Re}\left[\left(P_{0} u, u\right)_{0}+(\lambda u, u)_{0}\right] \geq \operatorname{Re}\left[\sum_{i=0}^{n-1}\left(b_{i} u^{(i)}, u\right)_{0}\right. \\
\left.\quad+\sum_{i=n}^{2 n-3}\left(u^{(n-1)},\left(\bar{b}_{i} u\right)^{(i-n+1)}\right)_{0}(-1)^{i-n+1}\right]-\left|\left(u^{(n-1)}, P_{n-2} u\right)_{0}\right| \\
+\delta_{1}\left\|u^{(n-1)}\right\|_{0}^{2}+\left.\frac{(-1)^{n-1}}{2}\left|u^{(n-1)}\right|^{2} b_{2 n-1}\right|_{0} ^{T}+\sum_{i=1}^{4} \widetilde{F}_{i} u{\widetilde{\widetilde{U}_{i}} u+\lambda\|u\|_{0}^{2} .}^{2}
\end{aligned}
$$

Выражения вида $\left(u^{(n-1)}, \bar{b}_{i}^{(l)} u^{(k)}\right)_{0}, k<n-1, i \geq n,\left(u^{(i)} b_{i}, u\right)$, входящие в первое и второе слагаемые, оцениваются одинаково. Применяя неравенство Гёльдера, имеем

$$
\left|\left(u^{(n-1)}, \bar{b}_{i}^{(l)} u^{(k)}\right)_{0}\right| \leq\left\|u^{(n-1)}\right\|_{0}\left\|\bar{b}_{i}^{(l)} u^{(k)}\right\|_{0} .
$$

Последний множитель оцениваем через $\left\|b_{i}^{(l)}\right\|_{0}\left\|u^{(k)}\right\|_{L_{\infty}(0, T)}$. В силу вложения $W_{2}^{s}(0, T) \subset L_{\infty}(0, T)$ при $s>1 / 2[15$, теорема 4.6.1] последняя норма оценивается через $c\|u\|_{W_{2}^{s+n-2}(0, T)}, s \in(1 / 2,1)$, фиксировано. Таким образом,

$$
\left|\left(u^{(n-1)}, \bar{b}_{i}^{(l)} u^{(k)}\right)_{0}\right| \leq c_{0}\left\|u^{(n-1)}\right\|_{0}\|u\|_{W_{2}^{s+n-2}(0, T)} .
$$

Используя интерполяционное неравенство вида [15]

$$
\|u\|_{W_{2}^{s+n-2}(0, T)} \leq c\|u\|_{W_{2}^{n-1}(0, T)}^{\theta}\|u\|_{0}^{1-\theta}, \quad \theta(n-1)=s+n-2,
$$


получим

$$
\left|\left(u^{(n-1)}, \bar{b}_{i}^{(l)} u^{(k)}\right)_{0}\right| \leq c_{1}\|u\|_{0}^{1-\theta}\|u\|_{W_{2}^{n-1}(0, T)}^{1+\theta} .
$$

Оставшиеся слагаемые $\left(u^{(i)} b_{i}, u\right)$ также допускают оценку

$$
\left|\left(u^{(i)} b_{i}, u\right)_{0}\right| \leq c_{2}\|u\|_{0}^{1-\theta}\|u\|_{W_{2}^{n-1}(0, T)}^{1+\theta} .
$$

Тогда неравенство (26) можно переписать в виде

$$
\begin{aligned}
J_{2} \geq \delta_{1}\left\|u^{(n-1)}\right\|_{0}^{2}-c_{3} \| & u\left\|_{0}^{1-\theta}\right\| u \|_{W_{2}^{n-1}(0, T)}^{1+\theta} \\
& +\left.\frac{(-1)^{n-1}}{2}\left|u^{(n-1)}\right|^{2} b_{2 n-1}\right|_{0} ^{T}+\operatorname{Re} \sum_{i=1}^{4} \widetilde{F}_{i} \overline{u \widetilde{U}_{i} u}+\lambda\|u\|_{0}^{2} .
\end{aligned}
$$

Рассмотрим случай (а) (см. (11)). Тогда $U_{0 p} u=\alpha_{p, n-1} u^{(n-1)}(0)$ и равенство $U_{p} u=0$ переписывается в виде

$$
u^{(n-1)}(0)=\sum_{j=0}^{n-2}\left(\tilde{\alpha}_{i, j} u^{(j)}(0)+\tilde{\beta}_{i, j} u^{(j)}(T)\right) .
$$

Отсюда имеем

$$
\begin{aligned}
\left.\frac{(-1)^{n-1} b_{2 n-1}}{2}\left|u^{(n-1)}\right|^{2}\right|_{0} ^{T} \geq \frac{(-1)^{n-1} b_{2 n-1}(T)}{2}\left|u^{(n-1)}(T)\right|^{2} \\
\quad-c_{2} \sum_{j=0}^{n-2}\left(\left|u^{(j)}(0)\right|^{2}+\left|u^{(j)}(T)\right|^{2}\right) .
\end{aligned}
$$

Аналогично

$$
\begin{aligned}
\operatorname{Re} \sum_{i=1}^{4} \widetilde{F}_{i} u \overline{\widetilde{U}_{i} u} \geq-c_{3}\left|u^{(n-1)}(T)\right| \sum_{j=0}^{n-2}\left(\left|u^{(j)}(0)\right|\right. & \left.+\left|u^{(j)}(T)\right|\right) \\
& -c_{4} \sum_{j=0}^{n-2}\left(\left|u^{(j)}(0)\right|^{2}+\left|u^{(j)}(T)\right|^{2}\right) .
\end{aligned}
$$

Окончательно

$$
\begin{aligned}
J_{4}= & \left.\frac{(-1)^{n-1}}{2}\left|u^{(n-1)}\right|^{2} b_{2 n-1}\right|_{0} ^{T}+\operatorname{Re} \sum_{i=1}^{4} \widetilde{F}_{i} u \widetilde{\widetilde{U}_{i} u} \geq \frac{(-1)^{n-1}}{2} b_{2 n-1}(T)\left|u^{(n-1)}(T)\right|^{2} \\
& -c_{3}\left|u^{(n-1)}(T)\right| \sum_{j=0}^{n-2}\left(\left|u^{(j)}(0)\right|+\left|u^{(j)}(T)\right|\right)-c_{4} \sum_{j=0}^{n-2}\left(\left|u^{(j)}(0)\right|^{2}+\left|u^{(j)}(T)\right|^{2}\right) .
\end{aligned}
$$

Далее используем неравенство

$$
|a b| \leq \varepsilon|a|^{p} / p+|b|^{q} \varepsilon^{-q / p} / q, \quad 1 / p+1 / q=1, \varepsilon>0 .
$$

Отсюда

$$
J_{4} \geq \frac{\delta_{1}}{2}\left|u^{(n-1)}(T)\right|^{2}-\varepsilon\left|u^{(n-1)}(T)\right|^{2}-c(\varepsilon) \sum_{j=0}^{n-2}\left(\left|u^{(j)}(0)\right|^{2}+\left|u^{(j)}(T)\right|^{2}\right) .
$$


Как и выше, используя теоремы вложения, неравенства (27) и (32), получим

$$
J_{4} \geq \frac{\delta_{1}}{2}\left|u^{(n-1)}(T)\right|^{2}-\varepsilon\left|u^{(n-1)}(T)\right|^{2}-\varepsilon_{1}\|u\|_{W_{2}^{n-1}(0, T)}^{2}-c\left(\varepsilon, \varepsilon_{1}\right)\|u\|_{0}^{2} .
$$

Используя (33) в (29) вместе с неравенством (32), имеем

$$
\begin{aligned}
J_{2} \geq \delta_{1}\left\|u^{(n-1)}\right\|_{0}^{2}+ & \frac{\delta_{1}}{2}\left|u^{(n-1)}(T)\right|^{2}+\lambda\|u\|_{0}^{2} \\
& -\varepsilon\left|u^{(n-1)}(T)\right|^{2}-\left(\varepsilon_{1}+\varepsilon_{2}\right)\|u\|_{W_{2}^{n-1}(0, T)}^{2}-c\left(\varepsilon, \varepsilon_{1}, \varepsilon_{2}\right)\|u\|_{0}^{2} .
\end{aligned}
$$

Не умаляя общности, считаем, что вместо стандартной нормы в $W_{2}^{n-1}(0, T)$ в предыдущем неравенстве используется эквивалентная норма $\left(\left\|u^{(n-1)}\right\|_{0}^{2}+\|u\|_{0}^{2}\right)^{1 / 2}$. Выбрав малые параметры $\varepsilon, \varepsilon_{1}, \varepsilon_{2}$ и достаточно большой параметр $\lambda_{0}$, для $\lambda \geq$ $\lambda_{0}$ получим неравенство

$$
J_{2} \geq \delta_{2}\left(\|u\|_{W_{2}^{n-1}(0, T)}^{2}+\left|u^{(n-1)}(T)\right|^{2}+\lambda\|u\|_{0}^{2}\right),
$$

которое гарантирует утверждение в случае (а). Здесь постоянная $\delta_{2}>0$ не зависит от $u$. Рассмотрение остальных случаев проходит совершенно аналогично, и их опустим. Приведем лишь предыдущую оценку в этих случаях. В случае (b) имеем

$$
\operatorname{Re}\left[\left(P_{0} u, u\right)_{0}+\left(\lambda_{0} u, u\right)_{0}\right] \geq \delta_{2}\left(\|u\|_{W_{2}^{n-1}(0, T)}^{2}+\left|u^{(n-1)}(0)\right|^{2}+\lambda\|u\|_{0}^{2}\right),
$$

а в случае (c) -

$\operatorname{Re}\left[\left(P_{0} u, u\right)_{0}+\left(\lambda_{0} u, u\right)_{0}\right] \geq \delta_{2}\left(\|u\|_{W_{2}^{n-1}(0, T)}^{2}+\left|u^{(n-1)}(0)\right|^{2}+\left|u^{(n-1)}(T)\right|^{2}+\lambda\|u\|_{0}^{2}\right)$,

Существование решений вытекает из известных результатов (см. [18]).

ЗАмЕчАниЕ 1. В некоторых случаях утверждение может быть доказано лишь при выполнении условия

$$
(-1)^{n-1}\left(b_{2 n-1}(T)-\alpha^{2} b_{2 n-1}(0)\right) \geq 0, \quad \alpha=\beta_{p, n-1} / \alpha_{p, n-1} .
$$

Например, это будет иметь место при условии, что $U_{p} u=u^{(n-1)}(0)-\alpha u^{(n-1)}(T)$ $=0$ и предпоследнее слагаемое в $(26)$ не содержит производных порядка $n-1$.

Приведем примеры коэрцитивных краевых условий.

Нелокальная задача. Рассмотрим условия

$$
\begin{gathered}
u^{(i)}(0)=\alpha u^{(i)}(T), \quad i=0,1, \ldots, 2 n-2, \alpha \neq 0, \alpha \in \mathbb{R}, \\
P_{2 n-1} u=\sum_{i=0}^{2 n-1} b_{i}(t) u^{(i)} .
\end{gathered}
$$

Утверждение теоремы 1 будет выполнено при выполнении условий (9), (10) и условий типа периодичности

$$
b_{i}^{(k)}(T)=\alpha^{2} b_{i}^{(k)}(0), \quad i \geq n, k \leq i-n+1 .
$$

В этом случае находимся в условиях предыдущего замечания и выражение (34) обращается в нуль. Естественно, такие операторы $P_{0}$ существуют, например, можем взять

$$
b_{2 n-1}=e^{\gamma t}, \quad \gamma=\frac{2}{T} \ln |\alpha|, \quad b_{2 n-2}=e^{\gamma t}\left((-1)^{n-1}+\gamma(n-1 / 2)\right),
$$


$b_{i}=0$ при $i<2 n-2$.

Задача Дирихле. $u^{(i)}(0)=u^{(i)}(T)=0, i=0,1, \ldots, n-2 ; u^{(n-1)}(0)=0$, если $(-1)^{n-1} b_{2 n-1}(T)>0 ; u^{(n-1)}(T)=0$, если $(-1)^{n-1} b_{2 n-1}(T)<0$. В качестве оператора $P_{2 n-1}$ можно взять оператор вида

$$
P_{2 n-1} u=\sum_{i=0}^{2 n-1} b_{i}(t) u^{(i)}
$$

удовлетворяющий условиям (9), (10).

Теорема 2. Пусть выполнены условия (9)-(12) для оператора

$$
P_{0} u=\sum_{i=0}^{2 n-1} b_{i} u^{(i)}
$$

и нормированного набора граничных операторов $U_{j}, j=1,2, \ldots, p$, порядков $k_{1} \leq k_{2} \leq \cdots \leq k_{p} \leq n-1$, причем соответствующие векторы $R_{i}, j=1,2, \ldots, p$, из коэффициентов операторов линейно независимы. Тогда найдутся граничные операторы $U_{p+1}, \ldots, U_{2 n-1}$ такие, что оператор $P_{0}$ с $D\left(P_{0}\right)=\left\{u \in W_{2}^{2 n-1}(0, T)\right.$ : $\left.U_{j} u=0, j=1,2, \ldots, 2 n-1\right\}$ коэрцитивен и справедливо утверждение теоремы 1.

ДокАЗАТЕЛЬСтво. Повторяя рассуждения, приведенные перед теоремой 1 , получим формулы (14), (15). Положим $U_{i}=F_{i+1}, i=p+1, \ldots, 2 n-1$. Легко видеть, что для нового набора граничных операторов $U_{i}, i=1,2, \ldots, 2 n-1$, будет выполнено условие (В). Далее применим теорему 1.

Теорема 2 определяет способ построения коэрцитивных граничных условий.

\section{3. Основные результаты}

Вместо уравнения (1) будем рассматривать уравнение с параметром вида

$$
L u=P u+M u+l u+\lambda u=f(x, t), \quad(x, t) \in Q=\Omega \times(0, T),
$$

где $\lambda$ - вещественный параметр,

$$
P=k_{2 n}(x, t) u^{(2 n)}+k_{2 n-1}(x, t) u^{(2 n-1)},
$$

$l$ - оператор вида

$$
l u=\sum_{i=0}^{n-1} \sum_{|\alpha|<m} k_{i, \alpha}(x, t) D^{\alpha} u^{(i)}+\sum_{i=n}^{2 n-2} \sum_{|\alpha|<m(1-(i-n+1) / n)} k_{i, \alpha}(x, t) D^{\alpha} u^{(i)} .
$$

Пусть выполнены условия

$$
\begin{gathered}
k_{i, \alpha}(x, t) \in L_{\infty}\left(0, T ; L_{p_{i}}(G)\right), \quad p_{i}>n /(m-|\alpha|) \quad \text { при } i<n, \\
\left.p_{i}>n /(m(1-(i-n+1) / n))-|\alpha|\right) \quad \text { при } i \geq n, k_{2 n-1}, k_{2 n}, \partial_{t} k_{2 n} \in L_{\infty}(Q) .
\end{gathered}
$$

Далее без ограничения общности считаем, что $M$ положительно определен, иначе заменим $M$ в (35) на $M+\lambda_{0}$. Определены операторы $M^{s}(s \in \mathbb{R})$. При наших предположениях на $Г$ и граничные операторы $B_{j}$ (следствие равенства $D\left(M^{s}\right)=\left(D(M), L_{2}(G)\right)_{1-s, 2}$, см. [15, п. 1.18.2] и описания пространства 
$\left(D(M), L_{2}(G)\right)_{\theta, 2}$ в $[19$, теорема $8.1 ; 20$, теорема 7.5$]$, см. также $[15$, п. 4.3.3]) имеем

$$
\begin{aligned}
D\left(M^{s}\right)=\left\{u \in W_{2}^{2 m s}(G):\left.B_{j} u\right|_{\Gamma}=0 \quad \text { для всех } j \text { с } m_{j}<2 m s-1 / 2,\right. \\
\\
\left.\int_{G} \frac{1}{\rho}\left|B_{j} u\right|^{2} d x<\infty \quad \text { при } m_{j}=2 m s-1 / 2\right\}, \quad \rho(x)=\operatorname{dist}(x, \Gamma) .
\end{aligned}
$$

Будем также использовать равенство (следствие равенств (6.8), (6.9) в [20])

$$
\left(W_{2}^{s}\left(0, T ; L_{2}(G)\right), L_{2}\left(0, T ; W_{2}^{r}(G)\right)\right)_{\theta, 2}=W_{2}^{s(1-\theta)}\left(0, T ; W_{2}^{r \theta}(G)\right), \quad \theta \in(0,1) .
$$

Положим $D\left(M^{s}\right)=\widetilde{W}_{2}^{2 m s}(G)$. Негативное пространство, построенное по $\widetilde{W}_{2}^{s}(G)$ и $L_{2}(G)$, обозначаем через $\widetilde{W}_{2}^{-s}(G)(s>0)$. Предположим, что выполнено условие

(C) Найдется дифференциальный оператор

$$
P_{0} u=\sum_{i=0}^{2 n-1} b_{i} u^{(i)}
$$

такой, что

$$
D\left(P_{0}\right)=\left\{u \in W_{2}^{2 n-1}(0, T): U_{j} u=0, j=1,2, \ldots, 2 n-1\right\},
$$

выполнены условия (9)-(12), (В) теоремы 1 , коэффициенты $k_{2 n}, k_{2 n-1}$ вещественны, $k_{2 n} b_{2 n-1}(T, x) \geq 0, k_{2 n} b_{2 n-1}(0, x) \leq 0$ в $G$ и найдется постоянная $\delta_{2}>0$ такая, что

$$
k_{2 n-1} b_{2 n-1}-\frac{1}{2}\left(k_{2 n} b_{2 n-1}\right)_{t}-k_{2 n} b_{2 n-2} \geq \delta_{2}>0, \quad\left|b_{2 n-1}\right| \geq \delta_{2} \quad \forall(x, t) \in Q .
$$

Теорема 3. Предположим, что выполнены условия (А), (C), (6), (7), (36), (37) и $f \in L_{2}(Q)$. Тогда найдется $\lambda_{1} \geq 0$ такой, что при $\lambda \geq \lambda_{1}$ существует решение задачи (35), (2), (3) такое, что

$$
u \in L_{2}\left(0, T ; \widetilde{W}_{2}^{(1-1 / 2 n) 2 m}(G)\right) \cap W_{2}^{2 n-1}\left(0, T ; L_{2}(G)\right) .
$$

ДокАЗАТЕЛЬСтво. Воспользуемся утверждением теоремы 1. Возьмем базис $\psi_{j}(t)$ в пространстве $W_{2}^{1}(0, T)$ и базис $\left\{\omega_{i}\right\}$ в пространстве $\widetilde{W}_{2}^{2 m}(G)$ из собственных функций оператора $M$, нормированных в $L_{2}(G)$. Тогда система $\left\{\psi_{j} \omega_{i}\right\}$ - базис в пространстве $W_{2}^{1}\left(0, T ; L_{2}(G)\right) \cap L_{2}\left(0, T ; \widetilde{W}_{2}^{2 m}(G)\right)$. Рассматривая оператор $P_{0}+\lambda_{0}$ вместо оператора $P_{0}$, если необходимо, без ограничения общности считаем, что выполнено неравенство (8) в случае $\lambda_{0}=0$ и справедливо утверждение теоремы 1 при $\lambda_{0}=0$.

Построим функции $\varphi_{j}(t)$ как решение уравнения $P_{0} \varphi_{j}=\psi_{j}$, удовлетворяющее краевым условиям (2). Ищем приближенное решение нашей задачи в виде

$$
u^{N}=\sum_{i, j=1}^{N} c_{i, j} \varphi_{j} \omega_{i},
$$

где постоянные $c_{i, j}$ определяем из системы уравнений

$$
\left(L u_{N}, \psi_{j} \omega_{i}\right)=\left(f, \psi_{j} \omega_{i}\right), \quad i, j=1, \ldots, N .
$$


Покажем разрешимость этой системы при подходящих $\lambda$. Умножим (39) на $c_{i, j}$ и просуммируем результат по $i, j$. Получим равенство

$$
\left(L u_{N}, P_{0} u_{N}\right)=\left(f, P_{0} u_{N}\right) .
$$

В силу теоремы 1 (считаем, что $\lambda>0$ ) имеем

$$
\begin{array}{r}
\operatorname{Re}\left(M u_{N}+\lambda u_{N}, P_{0} u_{N}\right)=\operatorname{Re}\left(M^{1 / 2} u_{N}, P_{0} M^{1 / 2} u_{N}\right)+\lambda \operatorname{Re}\left(u_{N}, P_{0} u_{N}\right) \\
\geq \delta_{1}\left(\left\|u_{N}\right\|_{W_{2}^{n-1}\left(0, T ; W_{2}^{m}(G)\right)}^{2}+\lambda\left\|u_{N}\right\|_{W_{2}^{n-1}\left(0, T ; L_{2}(G)\right)}^{2}\right),
\end{array}
$$

где $\delta_{1}-$ некоторая положительная постоянная. Рассмотрим выражение

$$
\begin{aligned}
\operatorname{Re}\left(P u_{N}, P_{0} u_{N}\right)= & \operatorname{Re}\left(P u_{N}, b_{2 n-1} u_{N}^{(2 n-1)}+b_{2 n-2} u_{N}^{(2 n-2)}\right) \\
& +\operatorname{Re}\left(P u_{N}, P_{0} u_{N}-b_{2 n-1} u_{N}^{(2 n-1)}-b_{2 n-2} u_{N}^{(2 n-2)}\right)=J_{1}+J_{2} .
\end{aligned}
$$

Для первого слагаемого, интегрируя по частям, в силу $(\mathrm{C})$ получим

$$
\begin{gathered}
J_{1} \geq \frac{\delta_{2}}{2}\left(\left\|\left.\sqrt{\left|k_{2 n}(x, T)\right|} u_{N}^{(2 n-1)}(x, T)\right|_{L_{2}(G)} ^{2}+\right\| \sqrt{\left|k_{2 n}(x, 0)\right|} u_{N}^{(2 n-1)}(x, 0) \|_{L_{2}(G)}^{2}\right) \\
\quad+\delta_{2}\left\|u_{N}^{(2 n-1)}\right\|^{2}+\operatorname{Re}\left(b_{2 n-1} u_{N}^{(2 n-1)}, b_{2 n-2} u_{N}^{(2 n-2)}\right) \\
-\operatorname{Re}\left(\left(k_{2 n} b_{2 n-2}\right)_{t} u_{N}^{(2 n-1)}, u_{N}^{(2 n-2)}\right)+\left.\operatorname{Re} k_{2 n} b_{2 n-2} u^{(2 n-1)} \overline{u^{(2 n-2)}}\right|_{0} ^{T} .
\end{gathered}
$$

Последние третье, четвертое и пятое слагаемые оцениваются однотипно, например, для четвертого имеем (используем включения $b_{2 n-2} \in W_{2}^{1}(0, T), k_{2 n}, k_{2 n t} \in$ $\left.L_{\infty}(Q)\right)$

$$
\begin{gathered}
\left|\operatorname{Re}\left(\left(k_{2 n} b_{2 n-2}\right)_{t} u_{N}^{(2 n-1)}, u_{N}^{(2 n-2)}\right)\right| \leq c\left\|u_{N}^{(2 n-1)}\right\|\left(\left\|u_{N}^{(2 n-2)}\right\|+\left\|b_{2 n-2}^{\prime} u_{N}^{(2 n-2)}\right\|\right) \\
\leq c_{1}\left\|u_{N}^{(2 n-1)}\right\|\left\|u_{N}^{(2 n-2)}\right\|_{L_{2}\left(G ; L_{\infty}(0, T)\right)} \leq c_{1}\left\|u_{N}^{(2 n-1)}\right\|\left\|u_{N}\right\|_{L_{2}\left(G ; W_{2}^{2 n-2+\delta}(0, T)\right)} \\
\leq c_{2}\left\|u_{N}\right\|_{L_{2}\left(G ; W_{2}^{2 n-1}(0, T)\right)}^{1+\theta}\left\|u_{N}\right\|^{1-\theta} \leq \varepsilon\left\|u_{N}^{(2 n-1)}\right\|^{2}+c(\varepsilon)\left\|u_{N}\right\|^{2},
\end{gathered}
$$

где $\theta(2 n-1)=2 n-2+\delta, \delta \in(1 / 2,1)$ и воспользовались интерполяционным неравенством, неравенством (32), вложением $W_{2}^{\delta}(0, T) \subset C([0, T])$ при $\delta>1 / 2$ и тем, что стандартная норма в $W_{2}^{2 n-1}(0, T)$ и норма $\|u\|=\left(\left\|u^{(2 n-1)}\right\|_{0}^{2}+\|u\|_{0}^{2}\right)^{1 / 2}$ эквивалентны. Оценка для третьего слагаемого записывается в том же виде. Запишем оценку для последнего слагаемого:

$$
\begin{gathered}
\left|\operatorname{Re} k_{2 n} b_{2 n-2} u_{N}^{(2 n-1)} \overline{u^{(2 n-2)}}\right|_{0}^{T} \mid \\
\leq c\left(\left|k_{2 n}(x, T) u_{N}^{(2 n-1)}(T) u^{(2 n-2)}(T)\right|+\left|k_{2 n}(x, 0) u_{N}^{(2 n-1)}(0) u^{(2 n-2)}(0)\right|\right) \\
\leq \varepsilon\left(\left|k_{2 n}(x, T)\right|\left|u_{N}^{(2 n-1)}(T)\right|^{2}+\left|k_{2 n}(x, 0)\right|\left|u_{N}^{(2 n-1)}(0)\right|^{2}\right) \\
+c(\varepsilon)\left(\left|u^{(2 n-2)}(T)\right|^{2}+\left|u^{(2 n-2)}(0)\right|^{2}\right)
\end{gathered}
$$


где $\delta \in(1 / 2,1)$. Выбрав малое положительное $\varepsilon$, приходим к оценке

$$
\begin{aligned}
J_{1} \geq \frac{\delta_{2}}{4}\left(\left\|\sqrt{\left|k_{2 n}(x, T)\right|} u_{N}^{(2 n-1)}(x, T)\right\|_{L_{2}(G)}^{2}+\right. & \left.\left\|\sqrt{\left|k_{2 n}(x, 0)\right|} u_{N}^{(2 n-1)}(x, 0)\right\|_{L_{2}(G)}^{2}\right) \\
& +\frac{\delta_{2}}{2}\left\|u_{N}^{(2 n-1)}\right\|^{2}-c_{3}\left\|u_{N}\right\|^{2} .
\end{aligned}
$$

Как и при оценке последнего слагаемого в $J_{1}$, интегрируя по частям в выражении $J_{2}$ и используя условия на коэффициенты и (32), легко получить неравенство

$$
\begin{aligned}
\left|J_{2}\right| \leq \varepsilon\left(\left|k_{2 n}(x, T)\left\|\left.u_{N}^{(2 n-1)}(T)\right|^{2}+\left|k_{2 n}(x, 0) \| u_{N}^{(2 n-1)}(0)\right|^{2}\right)\right.\right. & \\
& +\varepsilon\left\|u_{N}^{(2 n-1)}\right\|^{2}+c_{2}(\varepsilon)\left\|u_{N}\right\|^{2} .
\end{aligned}
$$

При подходящем выборе параметра $\varepsilon$ получим неравенство

$$
\begin{aligned}
\operatorname{Re}\left(P u_{N}, P_{0} u_{N}\right) & \geq \frac{\delta_{2}}{8}\left(\left\|\sqrt{\left|k_{2 n}(x, T)\right|} u_{N}^{(2 n-1)}(x, T)\right\|_{L_{2}(G)}^{2}\right. \\
+ & \left.\left\|\sqrt{\left|k_{2 n}(x, 0)\right|} u_{N}^{(2 n-1)}(x, 0)\right\|_{L_{2}(G)}^{2}+\left\|u_{N}^{(2 n-1)}\right\|^{2}\right)-c_{4}\left\|u_{N}\right\|^{2} .
\end{aligned}
$$

Оценим оставшиеся слагаемые в $(40)$ вида $\left(l u_{N}, P_{0} u_{N}\right)$. Сюда входят всевозможные слагаемые вида $\left(k_{i, \alpha} D^{\alpha} u_{N}^{(i)}, b_{k} u_{N}^{(k)}\right)=J$. Пусть $i \geq n$. Используя неравенство Гёльдера, получим

$$
|J| \leq\left(\int_{0}^{T}\left\|k_{i, \alpha}\right\|_{L_{2 p^{\prime}}(G)}^{2}\left\|D^{\alpha} u_{N}^{(i)}\right\|_{L_{2 q}(G)}^{2}\right)^{1 / 2}\left\|u_{N}^{(k)}\right\| .
$$

Возьмем $2 p^{\prime}=p_{i}\left(p_{i}-\right.$ постоянная из $\left.(37)\right)$. Пусть, например, $i \geq n$. Тогда для величины $q=p^{\prime} /\left(p^{\prime}-1\right)$ имеем неравенство $\left.q<n /(n-2 m(1-(i-n+1) / n)+2|\alpha|)\right)$. Можем найти $s<m(1-(i-n+1) / n)-|\alpha|$ такое, что $q=n /(n-2 s)$. Тогда из предыдущего неравенства и вложения $W_{2}^{s}(G) \subset L_{2 q}(G)$ получим оценку

$$
|J| \leq c\left\|D^{\alpha} u_{N}^{(i)}\right\|_{L_{2}\left(0, T ; W_{2}^{s}(G)\right)}\left\|u_{N}^{(k)}\right\| \leq c\left\|u_{N}^{(i)}\right\|_{L_{2}\left(0, T ; W_{2}^{s+|\alpha|}(G)\right)}\left\|u_{N}^{(k)}\right\| .
$$

Отметим, что $s+|\alpha|<m(1-(i-n+1) / n)$. Имеем интерполяционное неравенство [15]

$$
\begin{aligned}
& \|u\|_{W_{2}^{s+|\alpha|}(G)} \leq c\|u\|_{W_{2}^{m(1-(i-n+1) / n)}(G)}^{\theta}\|u\|_{L_{2}(G)}^{1-\theta}, \quad \theta m(1-(i-n+1) / n)=s+|\alpha|, \\
& \text { откуда } \\
& \qquad|J| \leq c\left\|u_{N}^{(i)}\right\|_{L_{2}\left(0, T ; W_{2}^{m(1-(i-n+1) / n)}(G)\right)}^{\theta}\left\|u_{N}^{(k)}\right\|\left\|u_{N}^{(i)}\right\|^{1-\theta}
\end{aligned}
$$

Из равенства (38) с $s=n$ и $r=m$ вытекает, что

$$
\left\|u_{N}^{(i)}\right\|_{L_{2}\left(0, T ; W_{2}^{m(1-(i-n+1) / n)}(G)\right)} \leq c\left(\left\|u_{N}\right\|_{W_{2}^{2 n-1}\left(0, T ; L_{2}(G)\right)}+\left\|u_{N}\right\|_{W_{2}^{n-1}\left(0, T ; W_{2}^{m}(G)\right)}\right) .
$$

Используя это неравенство в (44) вместе с интерполяционным неравенством

$$
\left\|u^{(j)}\right\| \leq c\|u\|_{W_{2}^{2 n-1}(G)}^{\theta}\|u\|^{1-\theta}, \quad \theta(2 n-1)=j,
$$

и неравенством (32) получим оценку

$$
|J| \leq \varepsilon\left(\left\|u_{N}^{(2 n-1)}\right\|_{L_{2}\left(0, T ; L_{2}(G)\right)}^{2}+\left\|u_{N}\right\|_{W_{2}^{n-1}\left(0, T ; W_{2}^{m}(G)\right)}^{2}\right)+c(\varepsilon)\left\|u_{N}\right\|^{2} .
$$


Слагаемые вида $\left(k_{i, \alpha} D^{\alpha} u_{N}^{(i)}, b_{k} u_{N}^{(k)}\right)$ с $i \leq n-1$ оцениваются существенно проще, и оценки опустим. Используя (41)-(43), (45) с достаточно малым параметром $\varepsilon$ и (40), получим неравенство

$$
\begin{gathered}
\operatorname{Re}\left(f, P_{0} u_{N}\right)=\operatorname{Re}\left(L u_{N}, P_{0} u_{N}\right) \geq \delta_{4}\left(\left\|u_{N}^{(2 n-1)}\right\|^{2}+\left\|u_{N}^{(n-1)}\right\|_{L_{2}\left(0, T ; W_{2}^{m}(G)\right)}^{2}\right. \\
\left.+\left\|\sqrt{\left|k_{2 n}(x, T)\right|} u_{N}^{(2 n-1)}(x, T)\right\|_{L_{2}(G)}^{2}+\left\|\sqrt{\left|k_{2 n}(x, 0)\right|} u_{N}^{(2 n-1)}(x, 0)\right\|_{L_{2}(G)}^{2}\right) \\
-c_{4}\left\|u_{N}\right\|_{L_{2}(G)}^{2}+\lambda\left\|u_{N}\right\|_{W_{2}^{n-1}(G)}^{2}
\end{gathered}
$$

где $\delta_{4}, \delta_{5}>0-$ некоторые постоянные. Пусть $\lambda_{0}=2 c_{4}$. Тогда при $\lambda \geq \lambda_{0}$ верно неравенство

$$
\begin{aligned}
& \operatorname{Re}\left(f, P_{0} u_{N}\right) \geq \operatorname{Re}\left(L u_{N}, P_{0} u_{N}\right) \geq \delta_{4}\left(\left\|u_{N}^{(2 n-1)}\right\|^{2}+\left\|u_{N}^{(n-1)}\right\|_{L_{2}\left(0, T ; W_{2}^{m}(G)\right)}^{2}\right. \\
&+\left\|\sqrt{\left|k_{2 n}(x, T)\right|} u_{N}^{(2 n-1)}(x, T)\right\|_{L_{2}(G)}^{2} \\
&\left.+\left\|\sqrt{\left|k_{2 n}(x, 0)\right|} u_{N}^{(2 n-1)}(x, 0)\right\|_{L_{2}(G)}^{2}\right)+\frac{\lambda}{2}\left\|u_{N}\right\|_{W_{2}^{n-1}(G)}^{2},
\end{aligned}
$$

которое также можно переписать в виде

$$
\begin{aligned}
& \|f\|^{2} \geq \delta_{5}\left(\left\|u_{N}\right\|_{L_{2}\left(0, T ; W_{2}^{2 n-1}(G)\right)}^{2}+\left\|u_{N}\right\|_{W_{2}^{n-1}\left(0, T ; W_{2}^{m}(G)\right)}^{2}+\lambda\left\|u_{N}\right\|_{W_{2}^{n-1}(G)}^{2}\right. \\
& \left.\quad+\left\|\sqrt{\left|k_{2 n}(x, T)\right|} u_{N}^{(2 n-1)}(x, T)\right\|_{L_{2}(G)}^{2}+\left\|\sqrt{\left|k_{2 n}(x, 0)\right|} u_{N}^{(2 n-1)}(x, 0)\right\|_{L_{2}(G)}^{2}\right) .
\end{aligned}
$$

Оценка (46) гарантирует, что найдется подпоследовательность $u_{N_{k}}$ такая, что для всех производных, входящих в правую часть (46), имеет место сходимость

$$
D^{\alpha} u_{N_{k}}^{(i)} \rightarrow D^{\alpha} u^{(i)}, \quad u_{N_{k}}^{(2 n-1)}(x, 0) \rightarrow u_{0}, \quad u_{N_{k}}^{(2 n-1)}(x, T) \rightarrow u_{T},
$$

где $|\alpha| \leq m, i=0,1,2, \ldots, 2 n-1, u \in W_{2}^{2 n-1}\left(0, T ; L_{2}(G)\right) \cap W_{2}^{n-1}\left(0, T ; W_{2}^{m}(G)\right)$ и последние две сходимости имеют место в весовых пространствах $L_{2, k_{2 n}(x, 0)}\left(G_{0}\right)$ и $L_{2, k_{2 n}(x, T)}\left(G_{T}\right)\left(G_{0}=\left\{x:\left|k_{2 n}(x, 0)\right|>0\right\}, G_{T}=\left\{x:\left|k_{2 n}(x, T)\right|>0\right\}\right)$ соответственно с нормами

$$
\|u\|^{2}=\left(\int_{G}\left|k_{2 n}(x, 0) \| u\right|^{2} d x\right)^{1 / 2}, \quad\|u\|^{2}=\left(\int_{G}\left|k_{2 n}(x, T) \| u\right|^{2} d x\right)^{1 / 2} .
$$

Рассмотрим равенство (39). Перепишем его в виде

$$
\begin{aligned}
& \quad\left(-u_{N_{k}}^{(2 n-1)},\left(\psi_{j} k_{2 n}\right)_{t} \omega_{i}\right)+\left(k_{2 n-1} u_{N_{k}}^{(2 n-1)}, \psi_{j} \omega_{i}\right) \\
& +\left(M^{1 / 2} u_{N_{k}}, M^{1 / 2} \psi_{j} \omega_{i}\right)+\left(l u_{N_{k}}+\lambda u_{N_{k}}, \psi_{j} \omega_{i}\right) \\
& +\left(k_{2 n}(x, T) u_{N_{k}}(x, T), \psi_{j}(T) \omega_{i}\right)-\left(k_{2 n}(x, 0) u_{N_{k}}(x, 0), \psi_{j}(0) \omega_{i}\right)=\left(f, \psi_{j} \omega_{i}\right),
\end{aligned}
$$

где $i, j=1, \ldots, N_{k}$. Умножая (47) на постоянные $\gamma_{i, j}$ и суммируя по $i, j$, получим

$$
\begin{aligned}
& \left(-u_{N_{k}}^{(2 n-1)},\left(v k_{2 n}\right)_{t}\right)+\left(k_{2 n-1} u_{N_{k}}^{(2 n-1)}, v\right)+\left(M^{1 / 2} u_{N_{k}}, M^{1 / 2} v\right)+\left(l u_{N_{k}}, v\right)+\left(\lambda u_{N_{k}}, v\right) \\
& \quad+\left(k_{2 n}(x, T) u_{N_{k}}(x, T), v(x, T)\right)-\left(k_{2 n}(x, 0) u_{N_{k}}(x, 0), v(x, 0)\right)=(f, v), \quad(48)
\end{aligned}
$$
где $v=\sum_{i, j=1}^{N} \gamma_{i, j} \psi_{j} \omega_{i}$. Переходя к пределу по $k$, приходим к интегральному тож-
деству

$$
\begin{gathered}
\left(-u^{(2 n-1)},\left(v k_{2 n}\right)_{t}\right)+\left(k_{2 n-1} u^{(2 n-1)}, v\right)+\left(M^{1 / 2} u, M^{1 / 2} v\right)+(l u, v)+(\lambda u, v) \\
+\left(k_{2 n}(x, T) u_{T}(x), v(x, T)\right)-\left(k_{2 n}(x, 0) u(x, 0), v(x, 0)\right)=(f, v),
\end{gathered}
$$


В силу произвольности постоянных $\gamma_{i, j}$ заключаем, что равенство (49) выполнено для всех функций $v \in L_{2}\left(0, T ; \widetilde{W}_{2}^{m}(G)\right) \cap W_{2}^{1}\left(0, T ; L_{2}(G)\right)$. Из определения обобщенной производной получаем, что существует обобщенная производная $\partial_{t}\left(k_{2 n} u^{(2 n-1)}\right) \in L_{2}\left(0, T ; \widetilde{W}_{2}^{-m}(G)\right)$. Более того, определены следы

$k_{2 n} u^{(2 n-1)}(x, T)=k_{2 n}(x, T) u_{T} \in L_{2}(G), \quad k_{2 n} u^{(2 n-1)}(x, 0)=k_{2 n}(x, 0) u_{0} \in L_{2}(G)$.

Тогда уравнение (35) выполняется в пространстве $L_{2}\left(0, T ; \widetilde{W}_{2}^{-m}(G)\right)$. Интегрируя (35) от нуля до $t$, получим

$$
\begin{aligned}
M v+\lambda v & =\int_{0}^{t} f(x, \tau) d \tau-\int_{0}^{t} l u(x, \tau)+\left(k_{2 n t}-k_{2 n-1}\right) u^{(2 n-1)}(x, \tau) d \tau \\
& -k_{2 n} u^{(2 n-1)}(x, t)+k_{2 n}(x, 0) u_{0}(x) \in L_{2}(Q), \quad v=\int_{0}^{t} u(x, \tau) d \tau,
\end{aligned}
$$

поскольку правая часть в (50) принадлежит $L_{2}(Q), v \in L_{2}\left(0, T ; W_{2}^{2 m}(G)\right)$ и справедлива оценка

$$
\|v\|_{L_{2}\left(0, T ; W_{2}^{2 m}(G)\right)}+\lambda\|v\| \leq c\|f\| .
$$

Так как $v \in W_{2}^{2 n}\left(0, T ; L_{2}(G)\right)$, согласно $(39) v^{(i)} \in L_{2}\left(0, T ; W_{2}^{2 m(1-i / 2 n)}(G)\right)$. В частности, $v^{\prime}=u \in L_{2}\left(0, T ; W_{2}^{2 m(1-1 / 2 n)}(G)\right)$. Тогда из (51) также имеем оценку

$$
\|u\|_{L_{2}\left(0, T ; W_{2}^{2 m(1-1 / 2 n)}(G)\right)}+\lambda^{1-1 / 2 m}\|u\| \leq c_{1}\|f\| .
$$

Из уравнения вытекает, что $\partial_{t}\left(k_{2 n} u^{(2 n-1)}\right) \in L_{2}\left(0, T ; \widetilde{W}_{2}^{-m / n}(G)\right)$.

Лемма 1 [21, лемма 1.2]. Пусть $k \in C^{1}([0, T]), a \in W_{\infty}^{1}(0, T)$, функции $k, a$ вещественны и найдется постоянная $\delta_{0}>0$ такая, что $a-\frac{1}{2}\left|k_{t}\right| \geq \delta>0$ для всех $t \in(0, T)$ и $u$ - обобщенное решение уравнения

$$
l_{0} u=k u_{t}+a u=f
$$

из $L_{2}(0, T)$, причем $f \in W_{2}^{1}(0, T)$. Тогда $u \in W_{2}^{1}(0, T), k u_{t} \in W_{2}^{1}(0, T)$.

Лемма 2. Пусть $k \in C^{1}(0, T), a \in W_{\infty}^{1}(0, T)$ и найдется постоянная $\delta_{0}>0$ такая, что $a-\frac{1}{2}\left|k_{t}\right| \geq \delta>0$ для всех $t \in(0, T), k(0) \leq 0, k(T) \geq 0$. Тогда обобщенное решение $u \in L_{2}(0, T)$ уравнения (53) существует и единственно и существует последовательность $u_{n} \in W_{2}^{1}(0, T), k u_{n t} \in W_{2}^{1}(0, T)$ такая, что $\partial_{t} k u_{n} \rightarrow \partial_{t} k u, u_{n} \rightarrow u, l_{0} u_{n} \rightarrow f$ в $L_{2}(0, T)$ при $n \rightarrow \infty$.

ДокАзАТЕЛЬСтво. Существование решения легко доказать, например, методом Галёркина, используя первую априорную оценку $\left(l_{0} u, u\right) \geq \delta_{0}\|u\|_{L_{2}(0, T)}^{2}$, справедливую для всех $u \in W_{2}^{1}(0, T)$. Обобщенное решение будет обладать свойствами

$\left(-u, k v_{t}\right)+\left(\left(a-k_{t}\right) u, v\right)=(f, v) \forall v \in W_{2}^{1}(0, T), v(0)=v(T)=0, k u \in W_{2}^{1}(0, T)$.

Предположим, что существует два решения $u_{1}, u_{2}$. Тогда их разность $\omega$ также обобщенное решение уравнения $l_{0} \omega=0$. По лемме $1 \omega \in W_{2}^{1}(0, T)$. Рассматривая равенство $\left(l_{0} \omega, \omega\right)=0$ и интегрируя по частям, получим $\omega=0$. Построим 
последовательность $f_{n} \in W_{2}^{1}(0, T)$ такую, что $f_{n} \rightarrow f$ в $L_{2}(0, T)$, и построим обобщенное решение $u_{n}$ уравнения

$$
l_{0} u_{n}=f_{n}
$$

из класса $u_{n} \in L_{2}(0, T), k u_{n} \in W_{2}^{1}(0, T)$. По лемме $1 u_{n} \in W_{2}^{1}(0, T)$. Умножим уравнение $(54)$ на $u_{n}$ и проинтегрируем по $(0, T)$. Получим неравенство $\left\|u_{n}\right\|_{L_{2}(0, T)} \leq c\left\|f_{n}\right\|_{L_{2}(0, T)}$, которое, будучи записанным для разности $u_{n}-u_{m}$, влечет существование функции $u \in L_{2}(0, T)$ такой, что $u_{n} \rightarrow u$ в $L_{2}(0, T)$. Из (54) вытекает также сходимость в себе $k u_{n}$ в $W_{2}^{1}(0, T)$ к некоторой функции $v \in W_{2}^{1}(0, T)$, совпадающей с $k u$.

Лемма 3. Пусть $k \in C^{1}(0, T), k(0) \leq 0, k(T) \geq 0, u \in L_{2}(0, T), \partial_{t} k u \in$ $L_{2}(0, T)$. Тогда справедливо равенство

$$
\operatorname{Re} \int_{0}^{T} k u_{t} u(t) d t \geq \int_{0}^{T} \frac{-1}{2}|u(t)|^{2} d t+\frac{1}{2}\left(k(T)\left|u_{n}(T)\right|^{2}-k(0)\left|u_{n}(0)\right|^{2}\right) .
$$

ДокАЗАТЕЛЬСтво. Рассмотрим функцию $a$, удовлетворяющую условиям лемм 1,2 , и положим $l_{0} u=f \in L_{2}(0, T)$. Построим последовательность $u_{n}$, удовлетворяющую условиям леммы 2. Имеем

$$
\operatorname{Re}\left(k u_{n t}, u_{n}\right)=\int_{0}^{T} \frac{-k_{t}}{2}\left|u_{n}\right|^{2}(t) d t+\frac{1}{2}\left(k(T)\left|u_{n}(T)\right|^{2}-k(0)\left|u_{n}(0)\right|^{2}\right) .
$$

Это неравенство, записанное для разностей $u_{n}-u_{m}$, влечет существование пределов чисел $\lim _{n \rightarrow \infty} u_{n}(0)=\alpha_{0}, \lim _{n \rightarrow \infty} u_{n}(T)=\alpha_{T}$ (если $k(T) \neq 0, k(0) \neq 0$ соответственно). Переходя к пределу, получим равенство

$$
\operatorname{Re}\left(k u_{t}, u\right)=\int_{0}^{T} \frac{-k_{t}}{2}|u|^{2}(t) d t+\frac{1}{2}\left(k(T)\left|\alpha_{T}\right|^{2}-k(0)\left|\alpha_{0}\right|^{2}\right) .
$$

Рассмотрим равенство $\left(l_{0} u_{n}, v\right)=\left(f_{n}, v\right), v \in W_{2}^{1}(0, T)$. Интегрируя по частям, имеем

$$
\int_{0}^{T}-u_{n}(t) k v_{t}+\left(a-k_{t}\right) u_{n} v d t+k(T) u_{n}(T)-k(0) u_{n}(0)=(f, v) .
$$

Переходя к переделу по $n$, получим

$$
\int_{0}^{T}-u(t) k v_{t}+\left(a-k_{t}\right) u v d t+k(T) \alpha_{T}-k(0) \alpha_{0}=(f, v) .
$$

В силу произвольности $v$ заключаем, что $u(T)=\alpha_{T}, u(0)=\alpha_{0}($ в случае $k(T) \neq$ 0 и $k(0) \neq 0$ если, например, $k(T)=0$, то слагаемое $k(T) \alpha_{T}$ полагается равным нулю).

Теорема единственности решений, полученных в теореме 3 , имеет место при некоторых дополнительных ограничениях. Например, это условия дополнительной гладкости коэффициентов уравнения или другие условия. В приведенной ниже теореме чтобы получить теорему единственности, предполагаем 
что коэффициенты оператора $P$ не зависят от $x$. При этом используем более слабые условия на коэффициенты оператора $l$. Итак, рассматриваем уравнение $(35)$, где будем предполагать, что $P=k_{2 n}(t) u^{(2 n)}+k_{2 n-1}(t) u^{(2 n-1)}$ и $l-$ оператор вида

причем

$$
l u=\sum_{i=0}^{2 n-2} \sum_{|\alpha|<2 m(1-(i+1) / 2 n)} k_{i, \alpha}(x, t) D^{\alpha} u^{(i)}
$$

$$
\begin{gathered}
k_{i, \alpha}(x, t) \in L_{\infty}\left(0, T ; L_{p_{i}}(G)\right), \quad p_{i}>n /(2 m(1-(i+1) / 2 n)-|\alpha|), \quad i \leq 2 n-2, \\
k_{2 n-1} \in L_{\infty}(0, T), \quad k_{2 n} \in C^{1}([0, T])
\end{gathered}
$$

Теорема 4. Предположим, что для $f \in L_{2}(Q)$ выполнены условия (A), (6), (7), (55), (56), (C), где дополнительно к (9) потребуем, чтобы $b_{2 n-1} \in C^{1}([0, T])$ при $n=1$. Тогда найдется число $\lambda_{1} \geq 0$ такое, что при $\lambda \geq \lambda_{1}$ существует единственное решение $u \in L_{2}\left(0, T ; \widetilde{W}_{2}^{(1-1 / 2 n) 2 m}(G)\right) \cap W_{2}^{2 n-1}\left(0, T ; L_{2}(G)\right)$ задачи $(35),(2),(3)$.

ДокАзАтЕльство. Сначала докажем утверждение в случае более простого уравнения вида

$$
L_{0} u=P u+M u+\lambda u=f .
$$

Повторяем рассуждения из доказательства теоремы 3. Приближенное решение $u_{N}$, определяемое при помощи равенства (39), где оператор $L$ заменен на $L_{0}$, удовлетворяет оценке (46), т. е.

$$
\left\|u_{N}\right\|_{L_{2}\left(0, T ; W_{2}^{2 n-1}(G)\right)}^{2}+\left\|u_{N}\right\|_{W_{2}^{n-1}\left(0, T ; W_{2}^{m}(G)\right)}^{2}+\lambda\left\|u_{N}\right\|_{W_{2}^{n-1}(G)}^{2} \leq c\|f\|^{2}
$$

при всех $\lambda \geq \lambda_{0}$ для некоторого $\lambda_{0}>0$, где постоянные не зависят от параметров $\lambda, N$. Переходя к пределу, получим, что решение $u$ также удовлетворяет оценке

$$
\|u\|_{L_{2}\left(0, T ; W_{2}^{2 n-1}(G)\right)}^{2}+\|u\|_{W_{2}^{n-1}\left(0, T ; W_{2}^{m}(G)\right)}^{2}+\lambda\|u\|_{W_{2}^{n-1}(G)}^{2} \leq c\|f\|^{2} .
$$

Согласно (52) предельное решение удовлетворяет оценке

$$
\begin{aligned}
\|u\|_{L_{2}\left(0, T ; W_{2}^{2 n-1}(G)\right)}^{2} & +\|u\|_{W_{2}^{n-1}\left(0, T ; W_{2}^{m}(G)\right)}^{2}+\lambda\|u\|_{W_{2}^{n-1}(G)}^{2} \\
& +\|u\|_{L_{2}\left(0, T ; W_{2}^{2 m(1-1 / 2 n)}(G)\right)}^{2}+\lambda^{2(1-1 / 2 m)}\|u\|^{2} \leq c\|f\|^{2} .
\end{aligned}
$$

Покажем единственность построенных решений. Считаем, что собственные функции $\left\{\omega_{i}\right\}$ нормированы в пространстве $L_{2}(G)$. Тогда коэффициенты Фурье $u_{i}$ решения $u$ в этом базисе суть решения задачи

$$
L_{i} u_{i}=P u_{i}+\left(\lambda_{i}+\lambda\right) u_{i}=f_{i}=\left(f, \omega_{i}\right)_{0}=f_{i}, \quad U_{j} u_{i}=0, \quad j=1,2, \ldots, 2 n-1 .
$$

Предполагая, что существуют два решения, находим, что коэффициенты Фурье $\psi_{i}$ разности решений удовлетворяют равенству

$$
P \psi_{i}+\left(\lambda_{i}+\lambda\right) \psi_{i}=0, \quad U_{j} \psi_{i}=0, \quad j=1,2, \ldots, 2 n-1 .
$$

Из уравнения вытекает, что существует обобщенная производная $\partial_{t}\left(k \psi_{i}^{(2 n-1)}\right) \in$ $L_{2}(0, T)$. Умножив равенство $(61)$ скалярно в $L_{2}(0, T)$ на $P_{0} \psi_{i}$, интегрируя по частям, используя при этом лемму 3 (по сути повторяем доказательство оценки (58)), получим оценку $\left(\lambda \geq \lambda_{0}\right)$

$$
\left\|\psi_{i}\right\|_{W_{2}^{2 n-1}(0, T)}^{2}+\left(\lambda+\lambda_{i}\right)\left\|\psi_{i}\right\|_{W_{2}^{n-1}(G)}^{2} \leq 0
$$


т. е. $\psi_{i}=0$. В силу произвольности $i$ заключаем что наши решения совпадают. Таким образом, определен оператор

$$
L_{0}^{-1}: L_{2}(Q) \rightarrow W_{2}^{2 n-1}\left(0, T ; L_{2}(G)\right) \cap L_{2}\left(0, T ; \widetilde{W}_{2}^{2 m(1-m / n)}(G)\right) .
$$

При этом справедлива оценка (59) для $u=L_{0}^{-1} f$. Перейдем к рассмотрению задачи $(35),(2),(3)$. Ее можно переписать в виде

$$
v+l L_{0}^{-1} v=f, \quad L_{0} v=u .
$$

Покажем, что оператор $R=l L_{0}^{-1}: L_{2}(Q) \rightarrow L_{2}(Q)$ является сжатием, если параметр $\lambda>0$ достаточно велик. Получим оценки. Пусть $J=k_{i, \alpha} D^{\alpha} u$. Как в доказательстве теоремы 3 ,

$$
\|J\| \leq\left(\int_{0}^{T}\left\|k_{i, \alpha}\right\|_{L_{2 p^{\prime}}(G)}^{2}\left\|D^{\alpha} u^{(i)}\right\|_{L_{2 q}(G)}^{2} d t\right)^{1 / 2} .
$$

Возьмем $2 p^{\prime}=p_{i}\left(p_{i}-\right.$ постоянная из (55)). По условию $p_{i}>n /(2 m(1-(i+$ $1) / 2 n)-|\alpha|)$. Тогда для величины $q=p^{\prime} /\left(p^{\prime}-1\right)$ имеем неравенство $q<n /(n-$ $4 m(1-(i+1) / 2 n)+2|\alpha|))$. Можно найти $s<2 m(1-(i+1) / 2 n)-|\alpha|$ такое, что $q=n /(n-2 s)$. Тогда из предыдущего неравенства и вложения $W_{2}^{s}(G) \subset L_{2 q}(G)$ получим оценку

$$
|J| \leq c\left\|D^{\alpha} u^{(i)}\right\|_{L_{2}\left(0, T ; W_{2}^{s}(G)\right)} \leq c_{1}\left\|u^{(i)}\right\|_{L_{2}\left(0, T ; W_{2}^{s+|\alpha|}(G)\right)} .
$$

Отметим, что $s+|\alpha|<2 m(1-(i+1) / 2 n)$. Имеем интерполяционное неравенство [15]

$$
\|u\|_{W_{2}^{s+|\alpha|}(G)} \leq c\|u\|_{W_{2}^{2 m(1-(i+1) / 2 n)}(G)}^{\theta}\|u\|_{L_{2}(G)}^{1-\theta}, \quad \theta 2 m(1-(i+1) / 2 n)=s+|\alpha|,
$$

откуда

$$
|J| \leq c\left\|u^{(i)}\right\|_{L_{2}\left(0, T ; W_{2}^{2 m(1-(i+1) / 2 n)}(G)\right)}^{\theta}\left\|u^{(i)}\right\|^{1-\theta} .
$$

Из равенства (39) вытекает, что

$$
\begin{aligned}
& \left\|u^{(i)}\right\|_{L_{2}\left(0, T ; W_{2}^{2 m(1-(i+1) / 2 n)}(G)\right)} \\
& \quad \leq c\left(\|u\|_{W_{2}^{2 n-1}\left(0, T ; L_{2}(G)\right)}+\left\|\int_{0}^{t} u d \tau\right\|_{L_{2}\left(0, T ; W_{2}^{2 m}(G)\right)}\right) .
\end{aligned}
$$

Кроме того,

$$
\left\|u^{(i)}\right\| \leq c\|u\|_{W_{2}^{2 n-1}\left(0, T ; L_{2}(G)\right)}^{i /(2 n-1)}\|u\|^{1-i /(2 n-1)} .
$$

Из последних двух неравенств вытекает оценка

$$
|J| \leq c\left(\|u\|_{W_{2}^{2 n-1}\left(0, T ; L_{2}(G)\right)}+\left\|\int_{0}^{t} u(x, \tau) d \tau\right\|_{L_{2}\left(0, T ; W_{2}^{2 m}(G)\right)}\right)^{\theta_{1}}\|u\|^{1-\theta_{1}}
$$

для некоторого $\theta_{1} \in(0,1)$. Суммируя подобные оценки, будем иметь

$$
\begin{aligned}
& \left\|l L_{0}^{-1} v\right\| \leq M\left(\left\|L_{0}^{-1} v\right\|_{W_{2}^{2 n-1}\left(0, T ; L_{2}(G)\right)}^{2}\right. \\
& \left.\quad+\left\|\int_{0}^{t} L_{0}^{-1} v(x, \tau) d \tau\right\|_{L_{2}\left(0, T ; W_{2}^{2 m}(G)\right)}\right)^{\theta_{2}}\left\|L_{0}^{-1} v\right\|^{1-\theta_{2}},
\end{aligned}
$$


где $M$ - некоторая постоянная, не зависящая от $u$, и параметр $\theta_{2}$ максимальный из параметров $\theta_{1}$, возникающих в различных оценках. Используя далее оценку (59), получим $\left\|l L_{0}^{-1} v\right\| \leq M_{1}\|v\| / \lambda^{\left(1-\theta_{2}\right)(1-1 / 2 n)}$. Выберем параметр $\lambda$ так, что $M_{1} / \lambda^{\left(1-\theta_{1}\right)(1-1 / 2 n)}<1$. Тогда уравнение $(62)$ будет иметь единственное решение в необходимом классе.

\section{ЛИТЕРАТУРА}

1. Олейник О. А., Радкевич Е. В. Уравнения второго порядка с неотрицательной характеристической формой. М.: ВИНИТИ, 1971. (Итоги науки. Сер. математика. Математический анализ).

2. Врагов В. Н. О постановке и разрешимости краевых задач для уравнений смешанно-составного типа // Математический анализ и смежные вопросы математики. Новосибирск: Ин-т математики им. С. Л. Соболева, 1978. С. 5-13.

3. Врагов В. Н. Краевые задачи для неклассических уравнений математической физики. Новосибирск: Ин-т математики им. С. Л. Соболева, 1983.

4. Терехов А. Н. Нелокальные краевые задачи для уравнений переменного типа // Неклассические уравнения математической физики. Новосибирск: Ин-т математики им. С. Л. Соболева, 1985. С. $148-158$.

5. Глазатов С. Н. Нелокальные краевые задачи для уравнений смешанного типа в прямоугольнике // Сиб. мат. журн. 1985. Т. 26, № 6. С. 162-164.

6. Джамалов С. З. О корректности нелокальных краевых задач для многомерного уравнения смешанного типа // Применение методов функционального анализа к неклассическим уравнениям математической физики. Новосибирск: Ин-т математики им. С. Л. Соболева, 1989. С. 63-70.

7. Каратопраклиева М. Г. Об одной нелокальной краевой задаче для уравнения смешанного типа // Дифференц. уравнения. 1991. Т. 27, № 1. С. 68-79.

8. Джамалов C. З. О корректности одной нелокальной краевой задачи с постоянными коэффициентами для уравнения смешанного типа второго рода второго порядка в пространстве // Мат. заметки СВФУ. 2017. Т. 24, № 4. С. 17-28.

9. Dzhamalov $S$. Z. The nonlocal boundary value problem with constant coefficients for the multidimensional equation of the mixed type of the second kind, the second order // J. Siber. Federal University. 2018. V. 11, N 4. P. 472-461.

10. Джамалов C. З. О гладкости решения одной нелокальной краевой задачи для многомерного уравнения смешанного типа второго рода второго порядка в пространстве Соболева // Журн. Средне-Волжского мат. о-ва. 2019. Т. 21, № 1. С. 24-33.

11. Егоров И. Е, Федоров В. Е. Неклассические уравнения математической физики высокого порядка. Новосибирск: ВЦ СО РАН, 1995.

12. Чуешев A. В. Об одном линейном уравнении смешанного типа высокого порядка // Сиб. мат. журн. 2002. Т. 43, № 2. С. 454-472.

13. Джамалов C. З. Об одной нелокальной краевой задачи для уравнения смешанного типа высокого порядка // Тез. докл. 7-й Междунар. конф. по математическому моделированию. Якутск: Изд-во СВФУ, 2014. С. 37-38.

14. Lions J. L., Magenes E. Non-homogeneous boundary value problems and applications. Berlin; Heildelberg; New-York: Springer-Verl., 1972. V. 1.

15. Трибель X. Теория интерполяции. Функциональные пространства. Дифференциальные операторы. М.: Мир, 1986.

16. Grisvard P. Équations différentielles abstraites // Ann. Sci. Éc. Norm. Supér. (4). 1969. V. 2, N 3. P. 311-395.

17. Denk R., Hieber M., Prüss J. R-boundedness, Fourier multipliers and problems of elliptic and parabolic type. Providence, RI: Amer. Math. Soc., 2003. (Mem. Amer. Math. Soc.; V. 166).

18. Наймарк М. А. Линейные дифференциальные операторы. М.: Наука, 1969.

19. Grisvard P. Caractérisation de quelques espaces d'interpolation // Arch. Rat. Mech. 1967. V. 25, N 1. P. 40-63.

20. Grisvard P. Commutativité de deux foncteurs d'interpolation at applications // J. Math. Pures Appl. 1966. V. 45. P. 143-206. 
21. Чуешев А. В. Оценки резольвенты для обыкновенных дифференциальных операторов смешанного типа // Мат. тр. 2000. Т. 3, № 1. С. 144-196.

Поступила в редакцию 19 декабря 2019 г.

После доработки 19 декабря 2019 г.

Принята к публикачии 19 февраля 2020 г.

Джамалов Сирожиддин Зухриддинович Институт математики им. В. И. Романовского АН РУз, пр. М. Улугбека, 81, Ташкент 100125, Узбекистан; Филиал Российского государственного университета нефти и газа им. И. М. Губкина в г. Ташкенте, ул. Дурмон Йули, 34, Ташкент 10125, Узбекистан siroj63@mail.ru

Пятков Сергей Григорьевич

Югорский государственный университет, ул. Чехова, 16, Ханты-Мансийск 628012;

Институт математики им. С. Л. Соболева СО РАН, пр. Академика Коптюга, 4, Новосибирск 630090

s-pyatkov@ugrasu.ru, pyatkov@math.nsc.ru 